Gdańsk 2019, Nr. 40

https://doi.org/10.26881/sgg.2019.40.02

Joanna Godlewicz-Adamiec

Warschauer Universität / Uniwersytet Warszawski

https://orcid.org/0000-0003-3025-6413

\title{
Rezeptionsstrategien der deutschen Romantiker und Umwandlungen der mittelalterlichen Quellen des Stoffes am Beispiel der Literarisierung des Melusinenmythos
}

\begin{abstract}
Geschichten von Feen und Meerjungfrauen zählen zu den poetischen Universalien. Der hier vorliegende Beitrag will die Forschungen zum Melusine/Undine-Motiv erweitern und einen neuen Akzent setzen. Der Fokus wird auf die Epochendifferenz im Hinblick auf die Mischwesen und ihre Funktion in der Narration gelegt. Ziel des interdisziplinär konzipierten Beitrags ist es, Differenzen und Konvergenzen in der Literarisierung des Melusinenstoffes im Mittelalter und in der Romantik darzustellen. Die Analyse wird auf den Zusammenhang zwischen den Texten und auf epochentypische Facetten eingehen. Gesucht wird sowohl nach unabhängigen Parallelen als auch nach möglichen Rezeptionsstrategien.
\end{abstract}

Schlüsselwörter: Melusine/Undine-Motiv, Mittelalter, Romantik, Rezeptionsstrategien

Reception strategies of the German Romanticists and transformation of the medieval source of inspiration using the example of the presentation of the Melusine myth. Histories of fairies and mermaids are among the poetic universals. The article aims to expand the research on the Melusine/Undine-motif and present it from a new perspective. The emphasis is placed on how the mythical creatures are presented in various literary and cultural periods. The interdisciplinary contribution aims to present differences and convergences in the presentation of the Melusine motif in the Middle Ages and the Romantic period. The analysis in the article will deal with the context of the texts and features for the two cultural periods. It is looking for independent parallels as well as possible reception strategies.

Keywords: Melusine/Undine-motif, Middle Ages, Romanticism, reception strategies

Geschichten von Feen und Meerjungfrauen zählen zu den poetischen Universalien: Sie sind weltweit und aus unterschiedlicher Zeit bekannt (Quast 2004: 83). Um die Mitte des 15. Jahrhunderts bezeugt der Prosaroman Melusine Thüring von Ringoltingens die Existenz einer hybriden Protagonistin, der Melusine, und stellt die Geschichte des Adelsgeschlechts Lusignan dar. Im erstmals 1853 publizierten Märchen Das Stuttgarter Hutzelmännlein von Eduard Mörike - der Geschichte einer Wassernixe, die im Blautopf lebt (Mörike 1918: 129) - erscheint eine ganze Fülle von Motiven, die für die Romantik wichtig sind. 
Vor allem ist die Geschichte im Mittelalter angesiedelt, wobei das Mittelalterbild in Mörikes Erzählung nicht viel mit dem idealisierten und verklärten Mittelalterbild der (Früh-)Romantik gemein hat, das in Novalis' Schrift Die Christenheit oder Europa aus dem Jahre 1799 zum Ausdruck kommt (Novalis 2013: 499). Bei Mörike fungiert das Mittelalter nicht als Zeichen einer „Goldenen Vorzeit“ (Vögele 2005: 90-91). Darüber hinaus wird im Märchen die Farbe Blau häufig verwendet, es wird in der Tiefe gesucht (in diesem Fall in einem See statt in einem Bergwerk), und es handelt sich um eine Geschichte einer Wasserfrau, in der das Melusine/ Undine-Motiv einen zentralen Platz einnimmt (Vögele 2005: 87-88).

In der Forschung zum Melusine/Undine-Motiv werden verschiedene Aspekte der Geschichte hervorgehoben. In der ästhetisch-monastischen Tradition wurzeln all diejenigen Auslegungen, die in den Sirenen fisch- und vogelähnlichen Mischwesen - den Teufel, den verderblichen Einfluss der Frau, die Verlockungen des Reichtums und des Wohlstands oder die als Satansknechte dargestellten Spielleute und Gaukler sehen. Im Umkreis der entstehenden ritterlich-höfischen Kultur konnten sie jedoch auch als aktuelle Anklagen gegen den neuen Gesellschafts- und Lebensstils des Adels verstanden werden (Neumeyer 1997: 24). Vertreter der Annales-Schule wie z. B. Jacques Le Goff betrachten die an der Wende vom 14. zum 15. Jahrhundert entstandenen französischen Melusinenromane als Dokumente volkstümlicher Imaginationen und verstanden den narrativen „Kern“ als Baustein einer kollektiven Mentalität (Steinkämper 2007: 47). Viele heutige Analysen des Stoffes orientieren sich an den jüngsten feministischen Forschungsergebnissen, während oft vergessen wird, dass das mittelalterliche normativ-rechtliche Verständnis des Geschlechterverhältnisses dem späteren diametral entgegengesetzt ist (ebd.: 88). Die Versuche, die moderne Liebesund Geschlechterproblematik, die für die Gestaltungen des Melusinenstoffes im 19. und 20. Jahrhundert zentral ist, ${ }^{1}$ in den vormodernen Variationen der Melusinengeschichte wiederzufinden, können eher als eine ahistorische „Rückprojektion“ späterer Interpretationen betrachtet werden (ebd.: 63).

Der Melusinenstoff wurde sowohl im Mittelalter als auch in der Romantik gern literarisiert, was zum Beispiel die mittelalterliche Melusine Thüring von Ringoltingens (1456), das bereits genannte Märchen Das Stuttgarter Hutzelmännlein von Eduard Mörike (1863), Ludwig Tiecks Sehr wunderbare Historie von der Melusina $(1800)^{2}$ oder die prominente romantische Wasserfrauen-Erzählung von Friedrich de la Motte Fouqué Undine (1811) zeigen. Ihre Bedeutung für die Literarisierung des Stoffes ist relevant, da sich alle späteren Bearbeitungen mit den von Fouqué vorgenommenen Motivveränderungen in der einen oder anderen Weise konfrontieren ${ }^{3}$ (Stuby 1992: 93). Der vorliegende Beitrag verweist auf unterschiedliche

\footnotetext{
1 Zu feministischen Diskursen um Melusinen- und Undinengestalten in der Literatur des 20. Jahrhunderts siehe u. a. die Studie von Nina Nowara-Matusik (Nowara-Matusik 2018: 9-29).

2 Die Sehr wunderbare Historie von der Melusina knüpft an die „Magelone“ an. Tieck war von diesen Stoffen eingenommen, was weitere Fragment gebliebene Texte beweisen (Prolog zur Magelone, Melusine-Fragment, Das Donauweib) (Paulin 1987: 57).

3 Das Thema scheint universal und transdisziplinär zu sein. De la Motte Fouqué war nicht nur Autor des Märchens, sondern auch des Librettos der romantischen „Zauberoper“ Undine, der sechsten und gleichzeitigletzten Oper E. T. A. Hoffmanns. Den Stoff zu seinem romantischen Kunstmärchen wie auch zum fünf Jahre später verfassten Libretto entnahm de la Motte Fouqué wahrscheinlich der Legende über die Wassergöttin aus Paracelsus Liber
} 
Aspekte der Melusine/Undine-Geschichte, die für das Mittelalter essenziell waren, sowie auf die Bedeutung der mittelalterlichen Texte als Quelle für die romantische Literatur. Zugleich stellt er einen Versuch dar, am Beispiel der ausgewählten romantischen Texte zu zeigen, wie diese besprochenen Motive umgestaltet und neu verwendet werden. Die Analyse wird auf die Relation der Texte und epochentypische Facetten eingehen. Der Fokus wird sowohl auf unabhängige Parallelen als auch auf mögliche Rezeptionsstrategien gelegt.

\section{Feen, Werwölfe, Mandragoren - Mischwesen im Mittelalter}

Das Mittelalter besaß ein anderes Verständnis vom Tier als die Neuzeit, da im Mittelalter auch Fabelwesen zu den Tieren gezählt wurden (Obermaier 2009: 1). Die Grenze zwischen einheimischen, exotischen und fantastischen Tieren wurde in dieser Zeit oft verwischt: Im mittelalterlichen Europa galten Kreaturen wie Drachen, Basilisken und Greifen als exotisch oder selten, aber realistisch, während Tiere in Bestiarien oft fantastische Merkmale oder ein ungewöhnliches Aussehen aufwiesen. Im Physiologus, einer frühchristlichen Naturlehre und allegorischen Auslegung einzelner Tiere, deren erste Überlieferungen aus dem 2. bis 4. Jahrhundert stammen, werden Eigenschaften von Tieren geschildert, die bis heute als typisch gelten (Szyrocki 1982: 16). Übernatürliche Wesen und Monster waren in der imaginären und ikonographischen Sphäre des Mittelalters allgegenwärtig, und ihre Herkunft war vielfältig. Einige - wie der Leviathan - stammten aus der Bibel, andere - wie die Hydra - aus der griechisch-römischen Mythologie, während viele weitere dem Osten entlehnt wurden (Le Goff/Truong 2006: 128).

In den Mythen vieler Völker findet man das Motiv der Schlacht der Drachen (Heinzle 1994: 23). Monster haben ihren Platz schon um 700 v. Chr. gefunden. In der Theogonie von Hesiod brachte Echidna - halb Schlange, halb Nymphe - einen höllischen Cerberus, eine vielköpfige Hydra und eine gefährliche Sphinx zur Welt. Bald erschienen zwar in der imaginären Sphäre Helden, die diese Bestien besiegen sollten, Monster nahmen aber zweifellos die menschliche Vorstellungskraft in Besitz. Bereits in Altägypten und Mesopotamien wurde die Existenz von Monstern als Tatsache angenommen, und es kann davon ausgegangen werden, dass Menschen damals indirekt an ihre Realität glaubten. Im Alten Testament lassen sich ebenfalls Beschreibungen von übernatürlichen Wesen finden - von Behemoth (Hi 40, 15) und Leviathan (Hi 41, 1-34). Die apokalyptische Beschreibung einer siebenköpfigen Bestie,

de Nymphis, Sylphis, Pygmaeis et Salamandris et de caeteris spiritibus. Mehr dazu siehe: Borkowska-Rychlewska 2007: 27-29. Auf die Version de la Motte Fouqués bezogen sich auch Bildende Künstler. So schuf der Schweizer Johann Heinrich Füssli zwischen 1819 und 1821 auf der Grundlage der Erzählung de la Motte Fouqués einen Undinen-Zyklus, der sieben Zeichnungen und ein Ölbild umfasste. 150 Jahre später knüpft Ingeborg Bachmann in ihrer Erzählung Undine geht an Fouqués Text an, die emanzipatorisches Potential enthält und in der feministischen Forschung der 1980er Jahre als (nicht explizite) Frauenliteratur interpretiert wurde. Einen Wandel des narrativen Paradigmas in dieser Erzählung, in der die weibliche Figur das Wort hat und die Geschichte aus ihrer Perspektive erzählt wird, analysiert Nina Nowara-Matusik 2018: 13-14. Bei Thüring ist Melusine zwar keine stille Gestalt, doch wird nur indirekt über ihre Äußerungen gesprochen (Thüring von Ringoltingen 1979: 18). 
die aus dem Meer steigt, wird beispielsweise in der Bamberger Apokalypse dargestellt. Die Miniatur der Handschrift (Bamberg, Staatsbibliothek, MS A. II. 42) stellt auf Folio 32v Johannes als Halbfigur hinter einem Wassersegment dar, dem eine riesige, pantherähnliche Bestie mit Löwenkopf und Bärentatzen entsteigt. Im Nacken des Monsters hängt der vordere von sechs kleinen Drachenköpfchen (Godlewicz-Adamiec 2017: 193-207). Monster erscheinen auch in der Beschreibung des Kampfes des hl. Gregors gegen den Drachen und in der über die hl. Marta, die den Drachen Taraska zähmt (Dell 2012: 6-8).

Wahrscheinlich hat keine andere Epoche Tiere so oft dargestellt wie das Mittelalter, in dem sie als bedeutendes Dekorationselement in Kirchen dienten und von Klerus und Gläubigen täglich betrachtet werden konnten (Pastoureau 2006: 32). Elemente der vorchristlichen Mythologie bilden dabei Verweise auf die alte Idee der Kontinuität zwischen Mensch und Tier. So lassen sich an den Fassaden mittelalterlicher Kirchen auch hybride Tierkreaturen wie z. B. die Fee Melusine finden, die halb als Frau, halb als Schlange dargestellt wird. Im 13. und 14. Jahrhundert verschmilzt die Melusine-Sage mit der Legende über die Anfänge der Familie Lusignan (Krzywy 2015: 11) und Zweige dieser Luxemburger Familie stellten am Ende des Mittelalters ein Juwel her, das Melusine als geflügelten Drachen im Badetrog darstellt (Pastoureau 2006: 269).

Aus den obigen Beispielen ergibt sich, dass die Grenzen zwischen Mensch und Tier im Mittelalter verwischt und nicht ganz fest waren; von Bedeutung waren hierbei auch Transformationen von Menschen in Tiere (Friedrich 2009: 125). Unter den fantastischen Kreaturen ist die Gruppe der Hybriden interessant, die sich aus Teilen verschiedener Lebensformen (Tieren, Pflanzen, Menschen) zusammensetzen (Duszyński 2012: 64). Hybride sind als Mischwesen bzw. Mischungen des Menschlichen und Animalischen zu verstehen, also als Gestalten, die unterschiedliche tierische Merkmale von symbolischer oder metaphorischer Bedeutung aufweisen können. Dabei kombinieren Chimären menschliche und tierische Elemente, während sich viele weitere Lebewesen aus Teilen verschiedener Tiere zusammensetzen, wobei deren menschliche Eigenschaften dann nur auf den Geist beschränkt sind (Dell 2012: 7).

\section{Wasserfrauen in der Kultur - der Sirenenmythos und die weibliche Gespaltenheit}

Chimären sind also meist als Kreuzung verschiedener Arten entstanden: Es handelt sich um Menschen mit Tierköpfen oder Tiere mit menschlichen Köpfen oder Torsi um Meerjungfrauen, Sphinxe, Zentauren oder speziell die Gestalt der Melusine als Frau mit einem Schlangen- oder Fischschwanz, den sie versteckt, um eine eheliche, mütterliche und soziale Rolle zu spielen (Le Goff/Truong 2006: 129). Der Sirenenmythos bildet eine zentrale motivische Konstante der abendländischen Kultur. Die Sirenen erscheinen schon in der antiken Literatur (Homers Odyssee) und Kunst. ${ }^{4}$ In der griechischen Mythologie war die Sirene ein weibliches

\footnotetext{
4 Siehe das Vasenbild mit einer Darstellung des Odysseus und der Sirenen (ca. 475-450 v. Chr.). Eine Abbildung ist u. a. im Buch Griechische Vasenmalerei: Auswabl hervorragender Vasenbilder zu finden (Furtwängler/ Reichhold/Huber 1924:Tafel 9).
} 
Fabelwesen, ein Mischwesen, das ursprünglich aus einer Frau und einem Vogel, später aus einer Frau und einem Fisch, bestand und durch seinen betörenden Gesang die vorbeifahrenden Schiffer anlockte, um sie zu töten. Was über die Begegnung des Odysseus mit den Sirenen bei Homer ungesagt bleibt, ruft - wie Ann Maria Stuby treffend beobachtet - dazu auf, den Raum mit eigenen Vorstellungen über den Kampf und die Liebe zwischen den Geschlechtern auszufüllen (Stuby 1992: 9). Im Sirenenstoff von Bedeutung sind die Sehnsucht nach Aufösung der Ich-Grenzen und die konstante Faszination des Wassers. In dieser Geschichte wird auch nach den sozialen, ideologischen und psychologischen Ursprüngen und Konsequenzen weiblicher Gespaltenheit gefragt. Untrennbar bleiben hierbei die Aspekte des Positiv-Sorgenden (Undine) und des Negativ-Zerstörerischen (Melusine) (ebd.: 1992: 13).

In den mittelalterlichen Texten kam es generell im Vergleich zur griechischen Mythologie, in der sich Psyche mit dem Gott Amor verbindet, generell zu einer Umkehrung des Geschlechterverhältnisses (Lundt 1991:30-31). In der theologischen und naturkundlichen Traktat-, Exempel- und Predigtliteratur des 12. und 13. Jahrhunderts finden sich zahlreiche Erzählungen, denen das Motiv der „gestörten Mahrtenehe “s als Erzähl- bzw. Strukturschema zugrunde liegt, und die sich als Variationen des Melusinenstoffes auffassen lassen, auch wenn die Protagonistinnen noch nicht die Bezeichnung Melusine tragen. Sie werden als „VorMelusinensagen" signiert (Steinkämper 2007: 64). Von der Popularität des Motivs im Mittelalter zeugt, dass dessen Verarbeitungen nicht nur aus dem 12./13. Jahrhundert bekannt sind, sondern dass sich eine weitere Welle von Texten in der späteren Phase der Epoche nachweisen lässt. Erst im Spätmittelalter erhält dieses weibliche Wesen den Namen „Melusine“. Im Laufe der Jahrhunderte erfährt das Motiv der gestörten Mahrtenehe eine sichtbare Umgestaltung: Der unkomplizierte Optimismus der Liebesphantasien des 12. Jahrhunderts weicht komplizierten Problemen, die eine unbefangene Zweisamkeit erschweren. Wie Bea Lundt aufweist, verstärkt die Christianisierung Ängste und führt zur Abwehr weiblicher Sinnlichkeit durch deren Dämonisierung und Verteufelung (Lundt 1991: 31-32). Auf die Bedrohung durch Sirenen weist beispielsweise der Physiologus Bis aus dem 13. Jahrhundert hin (Fizjolog BIs 2005). Nach dieser Quelle ist bei diesen Wesen nur der Nabel menschlich, der Rest des Körpers und das Aussehen bis hin zu den Füßen gleichen einem Vogel. Ihr Gesang sei so schön, dass sie durch die „hörbare Süße der Stimme“" Menschen anlocken und anziehen, die aus der Ferne herbeiströmen. Die außergewöhnliche Freude am Gesang bezaubert subtil ihre Ohren und Sinne, so dass sie einschlafen (Fizjolog BIs 2005: 48). ${ }^{6}$ Ein interessanter Übergang in den Darstellungen der Sirenen lässt sich am Beispiel des illustrierten Physiologus Bernensis (Bern, Burgerbibliothek, Cod. 318) aus dem 9. Jahrhundert beobachten. Dort erscheint die Sirene

5 Der Terminus der Mahrtenehe, der eine Verbindung eines Sterblichen mit einem überirdischen Wesen bedeutet, deren Dauer an die Beachtung eines Verbotes gebunden ist (Lecoutex 1980: 59), wurde erst Anfang des 20. Jahrhunderts definiert. Der Ursprung der Definition lässt sich mit dem mittelhochdeutschen Wort Mahrte (das die weibliche Form Mahr einnimmt) in Verbindung setzten. Zu den ersten Feengeschichten überhaupt gehören Lais von Marie de France (Lundt 1991: 41). Dem Mahrtenehe-Motiv bei de la Motte Fouqué hinsichtlich des Themas Christentum und Heidentum, aber auch dem Problem der Seele, ist eine interessante Studie von Andrey Kotin gewidmet (Kotin 2016: 71-82).

6 Die Gefahr, die mit dem Gesang verbunden ist, kommt im Werk Die Lorelei zum Ausdruck (Heine 1975: 207-208) 
(fol. 13v-14r) vergleichbar mit dem Physiologus, in einem Kapitel zusammengefasst mit den Zentauren. Im Text werden die Sirenen als im Meer angesiedelte und todbringende Wesen dargestellt. Sie halten die Vorüberfahrenden durch ihren Gesang auf und bewirken, dass sie ins Meer stürzen. Als Mischwesen besitzen sie nur einen menschlichen Oberkörper. Eine genauere Untersuchung zeigt, dass der Unterkörper der Sirenen aus dem geringelten Schwanz eines Reptils oder Fisches entsteht, was eine Abweichung von der Textvorlage bedeutet, in der die Sirene als Mischwesen mit menschlichem Oberkörper und dem Unterkörper eines Vogels beschrieben wird (Lund 1997: 65).

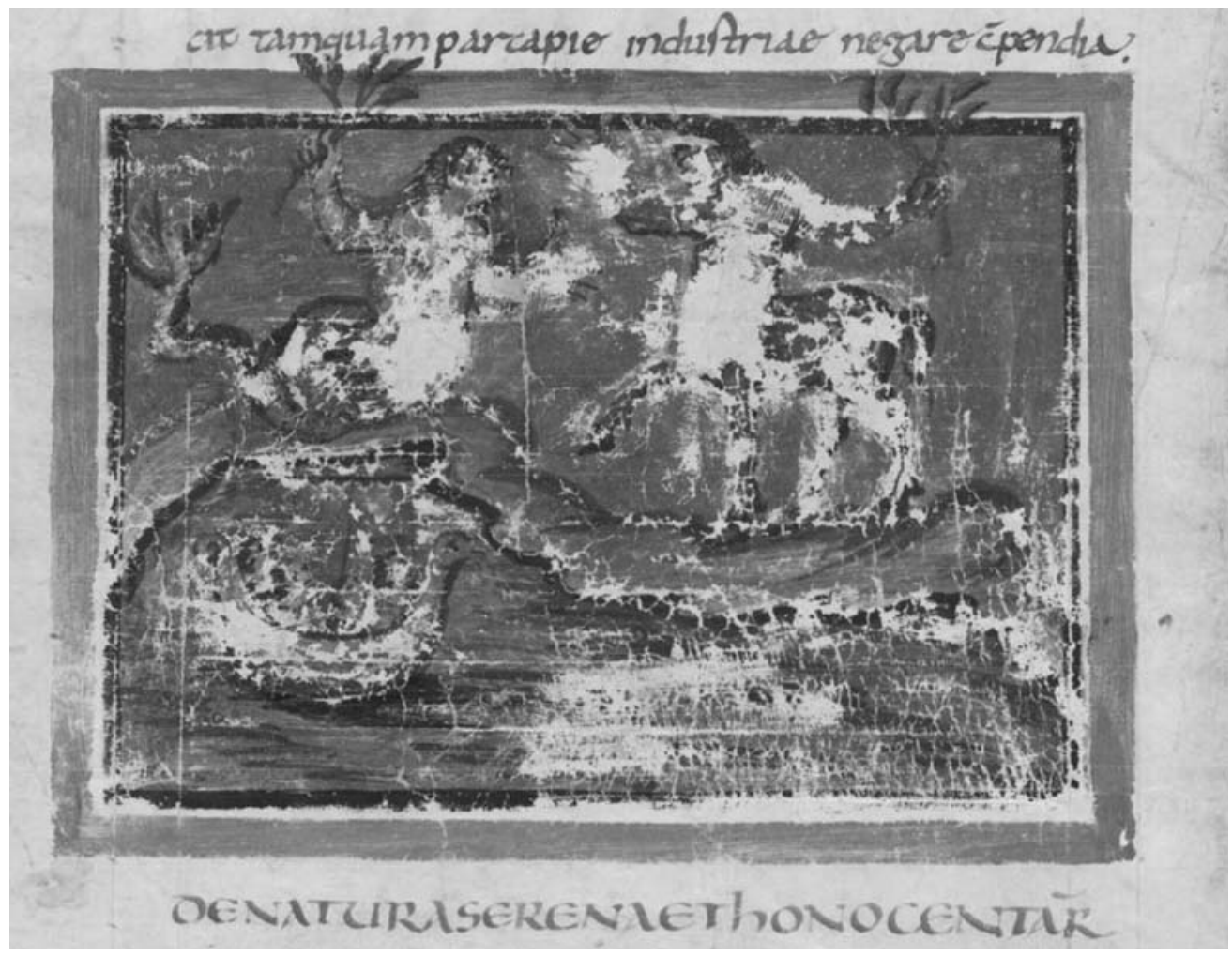

Abb. 1: Physiologus Bernensis, Bern, Burgerbibliothek, Cod. 318, https://www.e-codices.unifr.ch/en/list/ one $/ \mathrm{bbb} / 0318$, fol. $13 \mathrm{v}$.

Das Spätmittelalter widmet sich dem Motiv der Melusine in verschiedenen Varianten (Lundt 1991: 32). Der Perspektivenwandel, der den meisten Bearbeitungen des Stoffes im 19. Jahrhundert zugrunde liegt, wurde von Paracelsus' Aufspaltung des Weiblichen in die Aspekte des Positiv-Sorgenden (Undine) und des Negativ-Zerstörerischen (Melusine) eingeleitet. Diese Aspekte vermischen sich in der Ikonographie der Sirenen, Najaden und Nixen von der Antike bis ins Mittelalter. Von den deutschen Romantikern wurde die Melusinensage der Nachdichtung im Volksbuch des Thüring von Ringoltingen entnommen, während der Undine-Mythos der Staufenbergsage entstammt, die zu Beginn des 14. Jahrhunderts von Egenolf von Staufenberg in Verse gefasst wurde (Stuby 1992: 75). Das Melusinenmotiv wurde nicht nur in der 
Literatur (Friedrich de la Motte Fouqué, Ludwig Tieck, Eduard Mörike), sondern auch in den bildenden Künsten des 19. Jahrhunderts rezipiert, wie in der Darstellung der Melusine von Julius Hübner (Die schöne Melusine, 1844) oder in der Bilderreihe von Moritz von Schwind (Die schöne Melusine, 1869), einem der populärsten deutschen Spät-Romantiker mit seinen Gemälden zu Themen aus deutschen Sagen und Märchen.

\section{Die romantischen Varianten des Stoffes und ihre mittelalterlichen Quellen}

Der Erzählkern der Melusinengeschichten verbindet sich mit neuen Motiven, die etwas „über das Verhältnis des Autors zu den gesellschaftlich nicht integrierten oder integrierbaren Bestandteilen weiblicher Existenz und über männliche Wunschträume und Beziehungsphantasien seiner Zeit" (Lundt 1991: 115) aussagen. Die Verbindung eines Menschen mit einem jenseitshalbtierischen Wesen behandelte im 14. Jahrhundert der nordfranzösische Verfasser Jean d'Arras in seinem Prosaroman La noble histoire de Mélusine, dessen anderer Titel Le Roman de Mélusine en prose lautet. Um 1400 schrieb Couldrette einen Melusinenversroman mit dem Titel Le Roman de Mélusine ou Histoire de Lusignan. Thüring von Ringoltingens Prosaroman Melusine ist nach Couldrettes Vorlage verfasst (Quast 2004: 84; Steinkämper 2007: 73-74). Thüring von Ringoltingen mag es vorrangig darum gegangen sein, den Glanz und Vorbildcharakter französischer Lebens- und Hofkultur dem eigenen Kulturkreis einzuschreiben (vgl. dazu Dittenbass 2009: 62). Für diese Gruppe von längeren Erzähltexten in Prosa aus dem 15. und 16. Jahrhundert war in der Romantik der Begriff, „Volksbuch“ geprägt worden. Der Begriff ist jedoch irreführend hinsichtlich der im 15. und 16. Jahrhundert bestehenden literatursoziologischen Position der Texte, da für die Melusine sowohl der Autor als auch das Publikum nicht ,volkstümlich“ sind. In der neueren Forschung ist deshalb der Terminus „Prosaroman“ - in Abgrenzung zum höfischen Versroman des Hoch- und Spätmittelalters - üblich geworden (Steinkämper 2007: 81-82).

Thüring gestaltete eine neue Variante der Geschichte, die zur Grundlage für die Tradition der Melusine wird (Lundt 1991: 143). Die Melusine Thüring von Ringoltingens erzählt die Geschichte des Adelsgeschlechts Lusignan. Es ist eine Geschichte von Lügen, Geheimnissen, von Schuld und Verrat, von Liebe, Macht und Gewalt (Ziep 2006: 235). Gegenüber der Melusinenversion des Jean d'Arras treten in Thürings Bearbeitung die zauberischen und magischen Elemente stark zurück. Thüring nimmt an Melusine vor allem ihr Herausgehobensein und ihre Außergewöhnlichkeit gegenüber anderen Wesen wahr (Steinkämper 2007: 101). Bei der Entdeckung ihres Geheimnisses stellt sich heraus, dass Melusine ein Mischwesen ist:

Raimund sahe nun durch das Loch hinein und sahe, daß sein Weib und Gemahl in einem Bad nacket saß, und sie war von dem Nabel auf aus der Maßen und unaussprechlich ein schön weiblich Bilde, von Leib und von Angesicht unsäglichen schön. Aber von dem Nabel hinab, da war sie ein großer, langer, feindlicher Wurmesschwanz von blauer Lazur mit weißer silbern Farbe und darunter silbern Tröpflein gesprenget untereinander, als denn ein Schlang gemeiniglich gestalt ist. (Thüring von Ringoltingen 1979: 71$)^{7}$

\footnotetext{
7 In allen zitierten Passagen aus Thürings Melusine wird die originale Schreibweise beibehalten.
} 


\subsection{Die Gestalt der Melusine - Innere Wandlung und Selbstfindung}

In der Kultur spiegelt sich in den Tiergestalten das schwierige Verhältnis zum Körper und zur Geschlechtlichkeit der Frau (Lundt 1991: 31). Die Melusine zeigt eine Frau, die in vielen Bereichen des Lebens dominant ist (beispielsweise ihrem Mann Ratschläge gibt), das gewöhnliche Verhältnis der Geschlechter wird verkehrt, was nur deswegen möglich ist, weil sie keine gewöhnliche Frau, sondern ein übernatürliches Wesen ist (Steinkämper 2007: 122). ${ }^{8}$ Das Wasser als Element der Feen ist durch seine Ruhelosigkeit und Beweglichkeit geeignet, die Stimmung des inneren Aufbruchs zu neuen Ufern zu symbolisieren (Lundt 1991: 117-118).

Die deutschen Romantiker rezipieren die Melusinensage im Allgemeinen über ihre Nachdichtung der Version des Thüring von Ringoltingen, und den Undine-Mythos entnehmen sie der oben erwähnten Staufenbergsage (Der Ritter von Staufenberg, 1310) (Stuby 1992: 75). Peter von Staufenberg muss seinen Ausflug in die Feenwelt mit dem Leben bezahlen; der spielerische Umgang mit dem Motiv schlägt hier in bitteren Ernst um (Lundt 1991: 119). Mit dem Ritter von Staufenberg wird das Motiv der Mahrtenehe für die Narration einer Familien-Genealogie herangezogen (ebd.: 125).

Um die Wende des 18. zum 19. Jahrhundert erlebte das Motiv der Melusine eine regelrechte Renaissance, wenn es nicht sogar zu einer literarischen Mode wurde. Die Hinwendung der Romantiker zu Elementargeistern (zu denen in der Vier-Elemente-Lehre neben Erd-, Luftund Feuergeistern auch Wassergeister oder Undinen gehörten), insbesondere zu Wasserfrauen, hatte freilich vielfältige Ursachen und Gründe. In den Erzählungen über die Wasserfrauen kommt der Zauber eines magischen Naturverständnisses, einer Belebung der Elemente zum Ausdruck, verbunden mit Vorstellungen von einer übermächtigen oder dämonischen Weiblichkeit und weiblicher Naturverbundenheit. Mit dem Wasserfrauen-Motiv wird das Fremde und Bedrohliche, das Triebhafte sowie Abgründige und zugleich die innige Verbundenheit mit dem Elementaren verknüpft (Vögele 2005: 91-92).

In Ludwig Tiecks Sehr wunderbare Historie von der Melusine, die mit deutlicher Akzentverschiebung an die Magelone anknüpft und in die Romantischen Dichtungen aufgenommen wurde, mischt sich dem naiven Volkston auch eine Ebene der Erotik bei (Paulin 1987: 56-57). Tieck war in der Lage, eine altbekannte Figur wie die der mittelalterlichen Fee wiederzubeleben und sie neben elementare Geister, Goblins und allerlei fantastische Kreaturen zu stellen, die die Literatur dieser Zeit bewohnten. Tiecks Melusine lässt sie eher einer Wasserfrau als einer mittelalterlichen Fee ähneln.

8 Das Thema der Beziehung zwischen den Geschlechtern befand sich durchaus im Fokus der mittelalterlichen Verfasser. Konrad von Würzburg zeigt in seinem Werk Partonopier und Meliur (1277) die Zauberin Meliur nicht als gefährliche Hexe oder lockende Nixe, nicht einmal als Waldfee, sondern als Frau mit besonderen Fähigkeiten, die sich einen Freiraum für spielerische Experimente mit dem anderen Geschlecht schafft. Konrad thematisiert die Reifezeit der Protagonisten. Die Trennung der Geliebten stellt die Liebesbeziehung nicht in Frage, Partonopiers Mutter vertritt traditionelle Vorstellungen von der Partnerwahl. Ein Gegenmodell antizipiert eine Unabhängigkeit und die Privatheit der Verhältnisse. Die Versöhnung der Geliebten hängt von Meliurs Entscheidung und Partonopiers Entwicklung ab. Konrad von Würzburg gestaltet die persönlich-individuelle Entwicklung sowohl der männlichen als auch der weiblichen Psyche als Voraussetzung für eine reife und funktionierende Liebesbeziehung (Lundt 1991: 101-112). 
Paracelsus ' Lehre von den elementaren Geistern bildete die Hauptinspirationsquelle jener Autoren der Romantik, die dieses Thema aufgegriffen haben (Saporiti 2013: 69). In den Erzählungen der Romantik wird die Natur als Triebgrund und zugleich als sittliches Prinzip stilisiert. Dem Wasserfrauen-Motiv wird somit ein Spannungsverhältnis eingeschrieben, das zwei Pole "natürlich-unschuldig" und „fremdartig-bedrohlich" umfasst.

Diese Verknüpfungen sind auch in der romantischen Erzählung Undine de la Motte Fouqués (1811) zu finden, die in zwei Teile zerfällt: Im ersten Teil steht die Liebesbegegnung zwischen Ritter Huldbrand und der Wasserfee Undine, dazwischen liegen die Trauung und vor allem die Hochzeitsnacht (de la Motte Fouqué 1849: 59-76), die Undines Wesen und den Fortgang der Erzählung verändern (Stuby 1992: 79).

Das seelenlose Naturwesen Undine erlangt durch die Heirat mit dem Ritter eine unsterbliche Seele. Nach der Heirat vollzieht sich eine innere Wandlung Undines, und aus dem launenhaften Naturgeschöpf wird eine liebende und leidende Frau. In dieser Metamorphose vollzieht sich der Sündenfall der unbewussten Natur in die bewusste menschliche Existenz. Der Preis für die Seele ist das irdische Leid, weswegen Undines Geschichte unter den Menschen zu einer Leidensgeschichte wird. Undine bleibt eine Fremde, ihr gelingt nicht die Integration in die höfisch-kulturelle Welt (Vögele 2005: 92). De la Motte Fouqué erkennt gefühlsmäßig das Geschlechterdilemma seiner Zeit, den Widerspruch zwischen dem weiblichem Streben und den Verordnungen gesellschaftlicher Fremdbestimmung. Bei Fouqué ist der Wandel der Melusine zur empfindlichen und verletzbaren Frau als Mutter vollzogen, wobei auch in den nachfolgenden Adaptionen des Stoffes gerade diese Seite der Protagonistin hervorgehoben wird. Zu einer weiteren Motivverschiebung bei Fouqué gehört, dass Undines Wunsch nach einer Seele ein fremdbestimmter Wunsch ist. Wie Stuby beobachtet, geht es nicht um eine allgemein-menschliche Seele, sondern um eine spezifisch weibliche (Stuby 1992: 91-93). De la Motte Fouqué drückt die Sehnsucht nach Versöhnung der Geschlechter, nach einer glückhaften, wechselseitigen Liebe aus. Diese Utopie wird jedoch im Fortgang der Erzählung enttäuscht (ebd.: 85).

In Eduard Mörikes Historie von der schönen Lau (im Stuttgarter Hutzelmännlein) finden wir eine Binnen- und Rahmenhandlung, in der die Geschichte einer Selbstfindung erzählt wird (Vögele 2005: 88). Die Titelfigur hält sich am Anfang der Binnenerzählung im Blautopf auf (Mörike 1918: 129), der einen Naturraum repräsentiert. Die Wasserfrau bewegt sich vom Grund des Blautopfs in den Brunnen des Nonnenhofs, es ist die erste Exkursion der Lau aus der „Unterwelt“. Sie hat sich demgemäß an den Rand des Kulturraums vorgewagt. Die Aufwärtsbewegung, die sie vollzieht, ist durch einen Dreischritt gekennzeichnet. Die Stationen der Lau auf ihrem Weg nach oben bilden: Blautopf, Kellerbrunnen und Wohnräume (Vögele 2005: 67-69).

Zusammenfassend lässt sich sagen, dass diese Geschichten - von Thüring, Tieck, de la Motte Fouqué und Mörike - auf unterschiedliche Art und Weise das Verhältnis der Natur und der höfisch-kulturellen Welt und der Angehörigkeit der Melusine zu diesen Sphären thematisieren. Sie bilden unterschiedliche Varianten der Geschichte der Zugehörigkeit der Melusine zur höfischen Welt und der Selbstfindung der weiblichen Figur. Während in den mittelalterlichen Geschichten das Außergewöhnliche der Melusine in der dynastischen Darstellung der Familiengeschichte platziert ist, spiegeln die Geschichten der Romantik die Belebung 
der Figur wider, drücken den Wunsch nach einer Versöhnung der Geschlechter aus und zeigen die innerliche Umwandlung und Aufwärtsbewegung der weiblichen Figur auf.

\subsection{Die Figur des Ehemanns von Melusine - Tabu- und Eidbruch}

Viele Erzählungen bringen die enge Verbindung von Menschen mit Tieren oder halbtierischen Wesen zur Sprache. Die wahre Identität der Undinen muss verborgen bleiben, da deren Aufdeckung regelmäßig in eine Katastrophe mündet. Das Hochmittelalter kennt eine ungebrochene Begeisterung für diesen Erzähltypus. Gervasius von Tilbury erzählt in den Otia imperalia (1209/1214) die Geschichte einer Frau, die regelmäßig zu spät in die Kirche kam, und als ihr Gemahl sie mit Gewalt in der Kirche zurückhielt, flog sie als Drachenfrau davon. Es lässt sich dabei eine Parallele zur Darstellung der Melusine in der Buchillustration ziehen, die auf dem Bild mit einem Nixenschwanz und mit Flügeln durch das Fenster des Schlosses flieht. In der Regel haftet solchen Gestalten etwas Dämonisches an (Quast 2004: 83-84) (s. Abb. 2).

Bei Thüring von Ringoltingen wird das Tabu der Melusine, der Fluch der samstäglichen Verwandlung, nicht, wie häufig in Melusinengeschichten, einfach als gegeben hingenommen, sondern familiengeschichtlich als Bestrafung durch die Mutter plausibilisiert (Quast 2004: 95). Melusine ist in dieser Version weder Ehebrecherin noch Monster, weil Raimund diesen Zusammenhang nicht herstellt bzw. nicht herstellen kann. Der Schrekken ist eher Ausdruck der Unmöglichkeit einer Erkenntnis, denn was Raimund im Bad sieht, kann er nicht begreifen. Er muss das Gesehene und Geschehene erst (ein)ordnen (Ziep 2006: 249-250). Thürings Erzählbericht bezeugt die Existenz seiner hybriden Protagonistin Melusine und ihrer Eigenschaft als Stammmutter mächtiger Geschlechter. Ein solches Projekt, die Konstruktion der mythischen Abstammung einer adligen Familie, ist für mittelalterliche genealogische Entwürfe nicht ungewöhnlich. Die Eheschließung eines menschlichen Vorfahren mit einem andersweltlichen Mahr bewirkte dort die Konstituierung von Herrschaft und die Machtentfaltung des Geschlechts. Während die lateinischen Texte am theologisch-dämonologischen und naturkundlichen Diskurs und an der strikten Grenzziehung zwischen Menschen- und Dämonenwelt festhalten, zeigt sich in den oben erwähnten spätmittelalterlichen Romanfassungen eine grundlegende Verschiebung. In diesen Versionen wurde der genealogisch-dynastische Diskurs dominant (Steinkämper 2007: 50).

Bei d'Arras und Couldrette sieht sich die andersweltliche Fee um ihre Hoffnung und Erlösung doppelt betrogen, weil der Ehemann das gegen ihn errichtete Verbot zweimal missachtet, zunächst visuell und dann verbal (ebd.: 75). Die Bedrohung durch den Eidund Tabubruch folgt daraus, dass Melusine einen Mann finden muss, der bereit ist, ihre Abwesenheit am Samstag zu akzeptieren, was damals eine erhebliche Beschränkung der ehelichen Gewalt des Mannes über die Frau bedeutete (ebd.: 95). Der Ritter war sich jedoch der Bedrohung seiner familiären Position nicht bewusst, und als Repräsentant der höfischen Welt trägt ihm erst der Bruder die entsprechenden Gerüchte zu (ebd.: 111): 


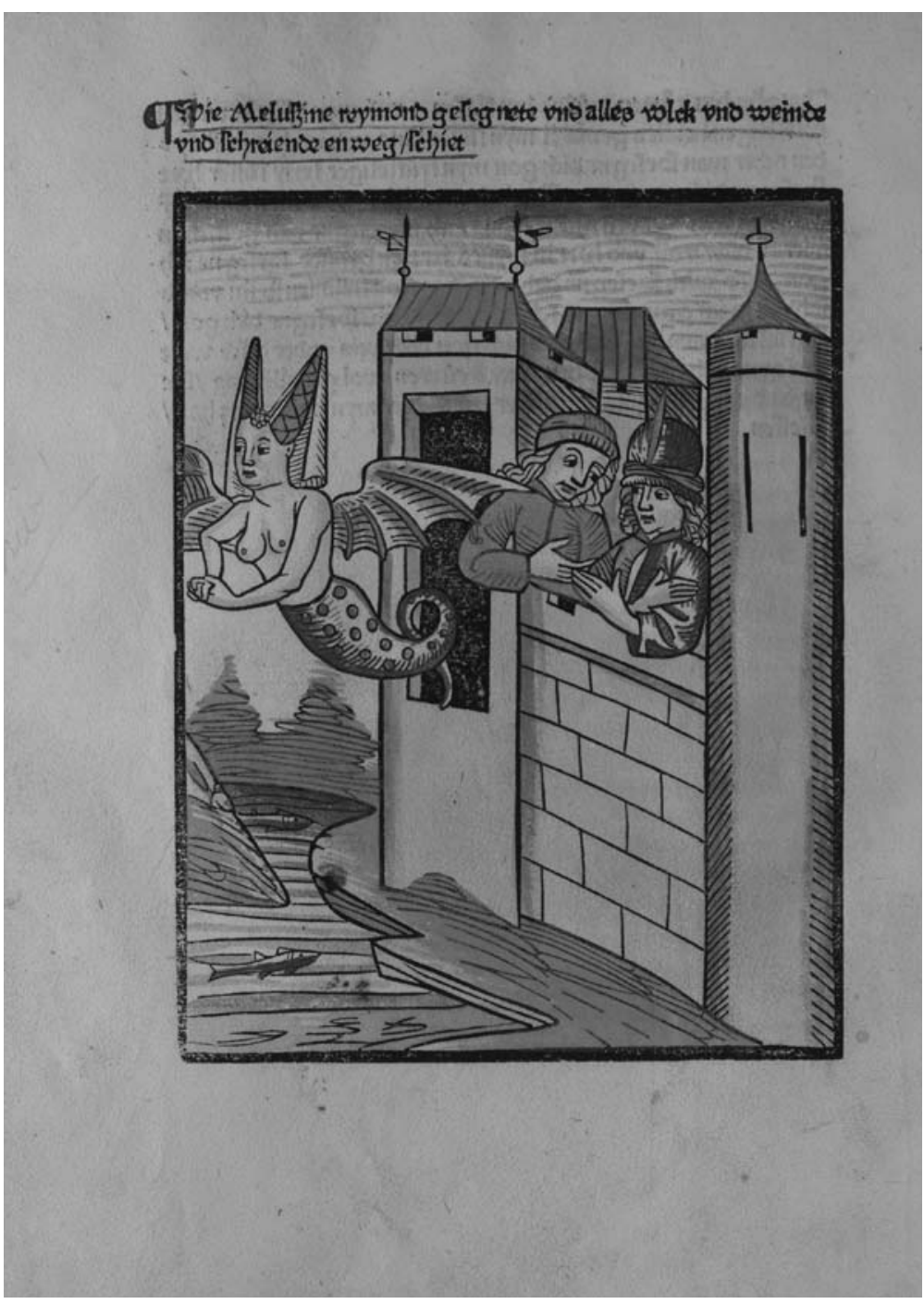

Abb. 2: Thüring von Ringoltingen, Melusine. Von einer frowen genant Melusina [Übersetzung und Neubearbeitung des Versromanes von Couldrette, ca. 1473/74], ULB Darmstadt, Inc IV 94, fol. 62v. Quelle: http://tudigit.ulb. tu-darmstadt.de/show/inc-iv-94/0126

Etliche sprechen, es sei ein Gespenst und ein Ungeheuerwesen um sie. Dies sag ich Euch als meinem lieben Bruder und rat Euch, daß Ihr gedenket zu wissen, was ihr Gewerb sei, daß Ihr nit zu einem Toren gemacht werdet und Ihr von ihr nit geäffet werdet. (Thüring von Ringoltingen 1979: 70)

Als Raimund „diese greusenliche und fremde Geschöpf an seinem Gemahl gesahe“ (ebd.: 72), gab es in seinem Herzen „großen Jammer und Herzensleid“ (ebd.: 73). Die Koppelung von Sicht- und Redeverbot, die erst zusammentreten müssen, um die Zerstörung der Beziehung zwischen Mensch und Mahr zu bewirken, scheint auf die hochmittelalterliche theologische 
Vorstellung zurückzugehen, nach der Dämonen nicht in den Menschen hineinsehen können. Thüring zeigte, dass Raimunds verbotener Blick auf die badende Melusine nicht genügte, da der Mann das Gesehene erst laut aussprechen musste, damit die Trennung unvermeidlich wurde. Es soll dabei betont werden, dass dieser Zusammenhang schon im 16. Jahrhundert nicht mehr verstanden wurde (ebd.: 119). Die Tabuverletzungen enden nicht mit der Bestrafung des Verletzenden, sondern mit der Entmachtung der tabuierten Person. Auf diese Art und Weise hat Raimunds unerlaubter Blick das dominante Feenwesen zur gewöhnlichen Ehefrau reduziert (ebd.: 115). Als er seine Frau im Bad überrascht und ihren Schlangenleib entdeckt, wird ihr Geheimnis endgültig enthüllt. Die Szene dieses Tabubruches wird nicht nur im Text, sondern auch in den ihm hinzugefügten Illustrationen reichlich geschildert. ${ }^{9}$

Für die Verarbeitung des Undine-Stoffes in der Romantik war die Verlagerung des Motivs kennzeichnend. Der Tabubruch des Ritters, der Undines Verschwinden aus der Menschenwelt zur Folge hatte, wurde nicht mehr durch eine zügellose Neugier motiviert, wie es in der mittelalterlichen Melusine der Ringoltingen-Sage noch der Fall war (die Frage, die die Handlung konfliktreich enden ließ, lautete: Was macht die Melusine samstags allein im Bade?), sondern von einer inneren Zerrissenheit des Ritters, die aus seiner aufkeimenden Liebe zu der Figur Bertalda erwächst. Naturkind Undine und Menschenfrau Bertalda stellen zwei Pole dar: die weiblich beseelte Naturhaftigkeit und die verbildete Weiblichkeit der Hofdamen. Das Epochenpaar der Romantik, Ritter und Wasser- bzw. Elementarwesen, muss also, um die vorzeitige Erlösung der Lebenden zu unterbinden, seine Liebe durch Treulosigkeit, Verrat und Tod beenden (Otto 2001: 68), was einen erheblichen Unterschied zu mittelalterlichen Geschichten in der Motivierung des Handlung und der Beziehungen zwischen den Figuren bildet.

Von dieser für die Romantik typischen Motivverlagerung bei der Aneignung des Undineoder Melusine-Stoffes ist der zweite Teil der Erzählung de la Motte Fouqués gekennzeichnet: Bei ihm wird der Tabubruch des Ritters Huldbrand nicht wie in den mittelalterlichen Textvorlagen mit der unbezähmbaren Neugier des Ehemannes, mit seinem Bedürfnis, ihr Geheimnis zu lüften und damit die Kontrolle auszuweiten, motiviert, sondern mit einer psychischen Verstrickung, die ihn als Opfer erscheinen lässt. Der Grund seines Misstrauens ist hier eher in der Neigung zu einer anderen Frau, Bertalda, zu suchen. Nicht ohne Bedeutung scheint es, dass die Protagonistinnen dieser Dreiecksbeziehung oppositionelle Züge tragen. Während der mittelalterliche Raimund Angst hatte, durch die wilde Naturhaftigkeit der Melusine um seine ritterliche Männlichkeit gebracht zu werden, ist der romantische Huldbrand zwischen zwei Welten und zwei Frauen zerrissen (Stuby 1992:79-80). Nach Stuby war de la Motte Fouqué der erste romantische Autor, der in den komplexen Motivverknüpfungen „ein elementares Bedürfnis seiner Epoche verspürte, das er in eine geeignete, ästhetische Form gießen wollte" (ebd.: 81).

Die romantischen Varianten des Stoffes und ihre mittelalterlichen Quellen unterschieden sich hinsichtlich der Motivation der Figuren, was besonders deutlich in der Tabu- und Eidbruchszene zum Ausdruck kommt. Man kann die Behauptung wagen, dass einige Motivationsgründe, die im Mittelalter relevant waren, in der neuzeitlichen Version unverständlich

9 Le Roman de Mélusine. Eins von sechzehn Bildern des Guillebert de Mets, circa 1410, Bibliothèque nationale de France. 
blieben. Hingegen versuchten die Autoren der Romantik, ihre Figuren um andere Motivationen zu bereichern.

\section{Resümee}

Die Erzählungen von eigenartigen Chimären haben sich seit Jahrtausenden verbreitet und tradiert. Einer der Gründe für ihre Existenz mag sein, dass diese Wesen das tiefe menschliche Bedürfnis nach dem Übernatürlichen befriedigen. Der Kampf zwischen Ordnung und Chaos sowie zwischen Gut und Böse wurde in den Götterkämpfen mit Chimären deutlich (Dell 2012: 7). Sie existieren, damit sich die Menschen ihnen stellen können, sie ermöglichen die innere Entwicklung und den Kampf mit den eigenen Schwächen (ebd.: 10). Die Anwesenheit von Chimären in der Welt der menschlichen Phantasie weist auf Ängste und Wünsche hin, ist Ausdruck ihrer komplexen Natur und ihrer inneren Dilemmata. Sie können Tabu-Grenzen und zwischenmenschliche Reaktionen reflektieren. Ihre Existenz und Besonderheiten berühren Fragen von universalem Charakter. Einen markanten Bereich der Kontakte des Menschen mit ihnen bilden die Mahrtenehen, mit deren Hilfe verschiedene Aspekte der Beziehungen zwischen Geschlechtern sowie unterschiedliche Ängste und Tabusphären thematisiert werden. Sowohl das Mittelalter als auch die Romantik haben ihre eigene spezifische Haltung zu Mischwesen gefunden, was sich am Beispiel der Literarisierung des Melusinenmythos zeigt. Der Stoff erhält in der Romantik neue Impulse und erfährt Perspektivverlagerungen.

Während die proto-melusinischen Erzählungen in der lateinischen Tradition als Beispiele für die Gefährlichkeit von Verbindungen mit Feenwesen fungierten, welche die Menschen durch Versprechungen an sich zu binden versuchten, entstanden fast 200 Jahre danach Romane, in denen die unheilvoll endende Liebesgeschichte zwischen einem Mann und einem andersweltlichen Mahr zu einer sich über mehrere Generationen erstreckenden Familiengeschichte wird (Steinkämper 2007: 73). Melusinas Schlangenschwanz erscheint in der mittelalterlichen Version nicht als Stigma, das sie für ihren Vatermord trägt, sondern als Zeichen, das sie mit ihrer Außergewöhnlichkeit zur Begründerin eines neuen Adelsgeschlechts, des Hauses Lusignan, prädestiniert.

Bei Thüring ist der Schlangenschwanz nicht Zeichen des Bösen, sondern eine von Gott erhaltene Besonderheit, die Melusine von anderen Wesen unterscheidet. Auf diese Weise wurde die andersweltliche Frau nicht mehr wie in den lateinischen proto-melusinischen Erzählungen des 12. Jahrhunderts als Dämon entlarvt und stigmatisiert, sondern in ihrer Seltsamkeit und Andersartigkeit bestaunt (Steinkämper 2007: 102-104).

Bei Thüring war die Meerfee von vornherein eine in die christliche Heilsgeschichte integrierte Figur, und das Dämonische war christlich absorbiert (Quast 2004: 90). Die Geschichte des Melusinen-Motivs zeigt, dass die Figur sogar mit dem Heiligtum assoziiert werden kann, was eines der Gemälde des Moritz von Schwind beweist. ${ }^{10}$ Wenn die Quellen der romantischen Versionen berücksichtigt werden, stellt sich heraus, dass Mischwesen und Mahrtenehen

10 Moritzvon Schwind, Die schöne Melusine: V: Das Heiligtum (1869), Aquarell aufKarton, $78 \times 133 \mathrm{~cm}$, https:// digital.belvedere.at/objects/12525/die-schone-melusine-ii-am-waldbrunnen/related/25/-1. Letzter Zugriff: 4.11.2019. 
ein universales Thema der Kultur bilden. Der Fokus wird in diesen Versionen freilich auf ganz andere Aspekte gelegt. Die Romantiker greifen nach dem Stoff, der sich im Mittelalter großer Beliebtheit erfreute, nutzen aber das darin steckende Potential, um andere menschliche Ängste, Hindernisse und Herausforderungen wie das Geschlechterdilemma, Misstrauen, Treulosigkeit, Zerrissenheit (zwischen zwei Welten/zwei Frauen) und Verrat zu thematisieren.

\section{Abbildungsverzeichnis}

1. Physiologus Bernensis, Burgerbibliothek Bern, Cod. 318, fol. 13v; https://www.e-codices.unifr.ch/ en/list/one/bbb/0318 [Foto: Codices Electronici AG, www.e-codices.ch].

2. Thüring von Ringoltingen, Melusine. Von einer frowen genant Melusina [Übersetzung und Neubearbeitung des Versromanes von Couldrette, ca. 1473/74], ULB Darmstadt, Inc IV 94, fol. 62v.; http://tudigit.ulb.tu-darmstadt.de/show/inc-iv-94/0126

\section{Literatur}

Borkowska-Rychlewska, Alina (2007): „Ondyna“ Hoffmanna - u źródeł romantycznej opery i dramatu [Hoffmanns „Undine”. An der Quelle der romantischen Oper und des Dramas]. In: Pamiętnik Literacki, XCVIII (2), 27-41.

Dell, Christopher (2012): Potwory. Bestiariusz przedziwnych stworzeń [Monster. Bestiarium fremder Kreaturen]. Warszawa: Wydawnictwo Albatros.

Dittenbass, Caterine (2009): Die „Melusine” des Thüring von Ringoltingen. Bezaubernde Worte - Gefährliches Schweigen. Zur Ambivalenz des Dialogs zwischen Reymond und Melusine in der Quellenszene. In: Barbara Fleith, René Wetzel (Hgg.): Kulturtopographie des deutschsprachigen Südwestens im späteren Mittelalter. Studien und Texte (= Kulturtopographie des alemannischen Raums, 1). Berlin: De Gruyter, 61-82.

Duszyński, Henryk (2012): Gryfy, harpie, syreny i inne stwory - o hybrydach w narracyjnych grach fabularnych [Griffe, Harpyien, Meerjungfrauen und andere Kreaturen - über Hybriden in narrativen Rollenspielen]. In: Homo Ludens. 1(4), 63-86.

Fizjolog BIs (2005). In: Stanisław Kobielus (Hg.): Fizjologi i Aviarium. Średniowieczne traktaty o symbolice zwierząt [Physiologe und Aviarium. Mittelalterliche Traktate über die Symbolik der Tiere]. Kraków: Wydawnictwo Benedyktynów Tyniec.

Fouqué, Friedrich de la Motte (1849): Undine. Eine Erzählung. Berlin: Ferd. Dümmler's Buchhandlung. Friedrich, Udo (2009): Menschentier und Tiermensch. Diskurse der Grenzziehung und Grenzüberschreitung im Mittelalter. Göttingen: Vandenhoeck \& Ruprecht.

Furtwängler, Adolf / Reichhold, Karl / Huber, Alois (1924): Griechische Vasenmalerei: Auswabl hervorragender Vasenbilder. München: F. Bruckmann.

Godlewicz-Adamiec, Joanna (2017): Monstra w literaturze i dekoracjach średniowiecznych katedr [Monster in der Literatur und den Dekorationen mittelalterlicher Kathedralen]. In: Miron Wolny, Małgorzata Chudzikowska-Wołoszyn (Hgg.): Paradoksologia w starożytności i średniowieczu 
[Paradoxologie in der Antike und im Mittelalter]. Olsztyn: Instytut Historii i Stosunków Międzynarodowych UWM, 193-207.

Heine, Heinrich (1975): Buch der Lieder. Heimkehr. In: Ders., Historisch-kritische Gesamtausgabe der Werke. Hg. von Manfred Widfuhr. Bd. 1.1. Hamburg: Hoffmann und Campe.

Heinzle, Joachim (1994): Das Nibelungenlied. Eine Einführung. Frankfurt a. M: Artemis.

Kotin, Andrey (2016): Die vertauschten Geschwister - Christenheit und Heidentum in Friedrich de la Motte Fouqués „Undine“. In: Studia Germanica Gedanensia (34), 71-82.

Krzywy, Roman (2015): Wstęp. In: Historia o szlachetnej a pięknej Meluzynie [Geschichte über die edle und schöne Melusine], bearb. von Roman Krzywy. Warszawa: Sub Lupa, 5-33.

Lecoutex, Claude (1980): Das Motiv der gestörten Mahrtenehe als Widerspieglung der menschlichen Psyche. In: Jürgen Janning u. a. (Hgg.): Vom Menschenbild im Märchen. Kassel: Röth, 59-71.

Le Goff, Jacques / Truong, Nicolas (2006): Historia ciata w średniowieczu [Orig.: Une histoire du corps au Moyen Age]. Aus dem Französischen übersetzt von Ireneusz Kania. Warszawa: Aletheia.

Lund, Cornelia (1997): Bild und Text in mittelalterlichen Bestiarien. In: Gisela Fabel, Georg Maag (Hgg.): Bestiarien im Spannungsfeld zwischen Mittelalter und Moderne. Tübingen: Narr, 62-74.

Lundt, Bea (1991): Melusine und Merlin im Mittelalter. Entwürfe und Modelle weiblicher Existenz im Beziebungs-Diskurs der Gescblechter. Ein Beitrag zur Historischen Erzählforscbung. München: Fink.

Mörike, Eduard (1918): Das Stuttgarter Hutzelmännlein. In: Ders., Gesammelte Werke. Bd. 3: Novellen und Märchen; Vermischte Schriften; Dramatisches. Hg. von Franz Deibel. Leipzig: Tempel Verlag.

Neumeyer, Martina (1997): Bestiaires - Überlieferung in neuerer Aktualität. In: Gisela Fabel, Georg Maag (Hgg.): Bestiarien im Spannungsfeld zwischen Mittelalter und Moderne. Tübingen: Narr, 15-28.

Novalis (2013): Werke. München: C. H. Beck.

Nowara-Matusik, Nina (2018): Ondyny i meluzyny we współczesnej niemieckojęzycznej literaturze kobiet (Bachmann, Neuwirth, Frischmuth) [Undinen und Melusinen in der deutschsprachigen Literatur der Gegenwart (Bachmann, Neuwirth, Frischmuth). In: Wortfolge/Szyk stów (2), 9-29.

Obermaier, Sabine (2009): Tiere und Fabelwesen im Mittelalter. Einführung und Überblick. In: Dies. (Hg.): Tiere und Fabelwesen im Mittelalter. Berlin/New York: De Gruyter, 1-25.

Otto, Beate (2001): Unterwasser-Literatur. Von Wasserfrauen und Wassermännern. Würzburg: Königshausen \& Neumann.

Pastoureau, Michel (2006): Średniowieczna gra symboli [Orig. Histoire symbolique du Moyen Âge occidental]. Aus dem Französischen übersetzt von Hanna Igalson-Tygielska. Warszawa: Oficyna Naukowa.

Paulin, Roger (1987): Ludwig Tieck. Stuttgart: Springer.

Quast, Bruno (2004): „Diß kommt von gelückes zuovalle“. Entzauberung und Remythisierung in der Melusine des Thüring von Ringoltingen. In: Ders., Udo Friedrich (Hgg.): Präsenz des Mythos: Konfigurationen einer Denkform in Mittelalter und Früher Neuzeit (= Trends in medieval philology, 2). Berlin/New York: De Gruyter, 83-98.

Saporiti, Sonia (2013): Myth as Symbol. A Psychoanalytic Study in Contemporary German Literature. Cambridge: Cambridge Scholar Publishing.

Steinkämper, Claudia (2007): Melusine - vom Schlangenweib zur, Beauté mit dem Fischschwanz: Geschichte einer literarischen Aneignung. Göttingen: Vandenhoeck \& Ruprecht.

Stuby, Ann Maria (1992), Liebe, Tod und Wasserfrau: Mythen des Weiblichen in der Literatur. Wiesbaden: Westdeutscher Verlag. 
Szyrocki, Marian (1982): Literatura niemieckiego obszaru językowego [Literatur des deutschen Sprachraums]. In: Władysław Floryan (Hg.): Dzieje literatur europejskich [Geschichte der europäischen Literaturen], Bd. 2. Warszawa: PWN, 11-235.

Thüring von Ringoltingen (1979): Die Historie von der schönen Melusina. Leipzig: Insel.

Tieck, Ludwig (1799-1800): Sehr wunderbare Historie von der Melusina. In drei Abteilungen. In: Ders., Romantische Dichtungen, Bd. 2. Jena: Friedrich Frommann, 331-464.

Vögele, Frank (2005): Leben als Hochseilakt. Studien zu Eduard Mörikes Erzählung „Das Stuttgarter Hutzelmännlein" (= Mannheimer Studien zur Literatur- und Kulturwissenschaft, 36). St. Ingebert: Röhrig Universitätsverlag.

Ziep, Franziska (2006): Geschlecht und Herkommen: Zur narrativen Struktur von Männlichkeit in der „Melusine“ des Thüring von Ringoltingen. In: Johannes Keller, Michael Mecklenburg (Hgg.): Das Abenteuer der Genealogie. Vater-Sohn-Beziehungen im Mittelalter (= Aventiuren, 2). Göttingen: $\mathrm{V} \& \mathrm{R}$ unipress, 235-262. 